\title{
A Comparison of Chest Radiograph and CTA Apical Pulmonary Findings in Patients Presenting with Suspected Acute Stroke during the COVID-19 Pandemic
}

I conjunction with our recently published article entitled "COVID-19 Stroke Apical Lung Examination Study: A Diagnostic and Prognostic Imaging Biomarker in Suspected Acute Stroke," ${ }^{1}$ the authors are pleased to also report our supplementary findings related to chest radiographs.

In our search for relevant diagnostic biomarkers within the lung apices on CTA performed in patients presenting with acute stroke, we also determined whether the chest radiograph is a reliable diagnostic imaging biomarker and how it compared with CTA-derived reliable diagnostic imaging biomarkers.

Chest radiographs are routine investigations for patients admitted with both suspected stroke and suspected coronavirus disease 2019 (COVID-19). The investigation is often available to a neuroradiologist when reviewing the CTA. In the setting of a neuroradiology department, we wished to determine whether the chest radiograph is a reliable diagnostic imaging biomarker (using the British Society of Thoracic Imaging COVID-19 Radiology Guidance; https://www.bsti. org.uk/standards-clinical-guidelines/clinical-guidelines/covid-19bsti-statement-and-guidance/) and how it compares with CTA-derived reliable diagnostic imaging biomarkers.

Anterior-posterior or posterior-anterior chest radiographs, if obtained within 6 hours of the CTA, were evaluated at the 3 sites included in our multicenter study in adult patients also undergoing CT of the head and craniocervical CTA as acute stroke investigations. Radiographs were acquired with standard techniques using digital radiography machines GC80 (Samsung), Discovery 656 (GE Healthcare), Multix Select (Siemens Healthineers), and Diagnost (Philips Healthcare).

Eighty-seven patients had a chest radiograph within 6 hours of the CTA; $55.2 \%$ (48/87) had normal findings; $13.8 \%(12 / 87)$ were COVID-19-typical; 26.4\% (23/87) had indeterminate findings; and 4.6\% (4/87) were non-COVID-19. By means of a descriptive COVID-19 chest radiograph grading system, the

This work was supported by the Wellcome/Engineering and Physical Sciences Research Council Center for Medical Engineering (WT 203148/Z/16/Z).

- Indicates open access to non-subscribers at www.ajnr.org

http://dx.doi.org/10.3174/ajnr.A6940 interrater reliability among the 4 neuroradiologist raters was "substantial" across the study (Fleiss $\kappa=0.73$; 95\% CI, 0.62-0.84). Shown to be reliable, we then determined the diagnostic accuracy of this chest radiograph imaging biomarker for Severe Acute Respiratory Syndrome coronavirus 2 (SARS-CoV-2) using the reverse transcriptase polymerase chain reaction (RT-PCR) test as the reference standard (Table). Fifty-nine patients had both SARSCoV-2 RT-PCR results and a chest radiograph. All measures of diagnostic accuracy of the chest radiograph were inferior to our selected CTA-derived reliable diagnostic imaging biomarkers.

Chest radiographs are routine investigations for those admitted with suspected stroke and those admitted with suspected COVID-19. In the setting of a neuroradiology department, while a descriptive chest radiograph grading system proved reliable, it was not an accurate COVID-19 diagnostic biomarker in the cohort of patients undergoing immediate CTA for suspected stroke, in contrast to the reliable diagnostic biomarkers identified in the CTA apical assessment.

Chest radiographs had a much lower diagnostic performance than all CTA biomarkers with a sensitivity of 50\% (95\% CI, 2971 ) and a specificity of $61 \%$ (95\% CI, 46-74) for COVID-19 (Table). We showed that chest radiograph assessment should not, therefore, replace a simple assessment of the presence of ground glass opacification on CTA for COVID-19.

Given the high frequency of chest radiographs as a screening investigation, particularly in the current era, with the additional prothrombotic sequalae of COVID-19, the findings are highly relevant to both emergency radiologists and neuroradiologists reporting acute stroke studies. Data pertaining to chest radiographs and COVID-19 in the acute stroke population have not been previously reported.

\section{REFERENCE}

1. Siddiqui J, Bala F, Sciacca S, et al. COVID-19 stroke apical lung examination study: a diagnostic and prognostic imaging biomarker in suspected acute stroke. AJNR Am J Neuroradiol 2020 Sept 17. [Epub ahead of print] CrossRef Medline

AJNR Am J Neuroradiol 42:E13-E14 Mar 2021 www.ajnr.org E13 
Diagnostic accuracy of chest radiograph and CTA features in patients who had undergone both a chest radiograph and a SARSCoV-2 RT-PCR test $(n=59)$

\begin{tabular}{|c|c|c|c|c|}
\hline & Sensitivity $(95 \% \mathrm{Cl})$ & Specificity $(95 \% \mathrm{Cl})$ & PPV $(95 \% \mathrm{CI})$ & NPV $(95 \% \mathrm{CI})$ \\
\hline COVID-typical/indeterminate (chest radiograph)a & $50 \%(29-71)$ & $61 \%(46-74)$ & $36 \%(24-51)$ & $74 \%(62-82)$ \\
\hline Presence of GGO (CTA) & $83 \%(58-96)$ & $80 \%(65-91)$ & $65 \%(43-83)$ & $92 \%(76-98)$ \\
\hline Focal GGO (CTA) & $56 \%(31-78)$ & $85 \%(70-94)$ & $62 \%(36-84)$ & $81 \%(66-91)$ \\
\hline Bilateral GGO (CTA) & $56 \%(31-78)$ & $83 \%(67-92)$ & $59 \%(33-81)$ & $80 \%(65-91)$ \\
\hline Peripheral GGO (CTA) & $78 \%(52-93)$ & $85 \%(70-94)$ & $70 \%(46-87)$ & $90 \%(75-97)$ \\
\hline COVID-typical/indeterminate (CTA) & $72 \%(46-89)$ & $90 \%(76-97)$ & $76 \%(50-92)$ & $88 \%(74-95)$ \\
\hline
\end{tabular}

Note:-PPV indicates positive predictive value; NPV, negative predictive value; GGO, ground glass opacification.

${ }^{a}$ COVID-typical alone gave a low sensitivity (44\%) and was excluded from further analysis.

\section{(D). Siddiqui}

(D) F. Bala

Department of Neuroradiology

King's College Hospital NHS Foundation Trust London, UK

(1)S. Sciacca

(1) A.M. Falzon

Lysholm Department of Neuroradiology

National Hospital for Neurology and Neurosurgery University College London Hospitals NHS Foundation Trust London, UK

(D) Menger Department of Neurology King's College Hospital NHS Foundation Trust London, UK

(D).A. Matloob Department of Neurosurgery National Hospital for Neurology and Neurosurgery University College London Hospitals NHS Foundation Trust London, UK

(1) F.N.A.C. Miller Department of Radiology King's College Hospital NHS Foundation Trust London, UK

(1) R.J. Simister Comprehensive Stroke Service University College London Hospitals NHS Foundation Trust
London, UK

Stroke Research Centre University College London Queen Square Institute of Neurology London, UK

(DI. Chatterjee Comprehensive Stroke Service University College London Hospitals NHS Foundation Trust London, UK

(D) L.K. Sztriha Department of Neurology King's College Hospital NHS Foundation Trust London, UK

(1). Davagnanam Lysholm Department of Neuroradiology National Hospital for Neurology and Neurosurgery University College London Hospitals NHS Foundation Trust London, UK

Brain Repair \& Rehabilitation Unit University College London Queen Square Institute of Neurology London, UK

(D) T.C. Booth

Department of Neuroradiology King's College Hospital NHS Foundation Trust London, UK

School of Biomedical Engineering and Imaging Sciences King's College London London, UK 